\title{
Efeito cicatrizante do extrato de plantago tomentosa em cadelas submetidas a ovariohisterectomia
}

\section{Cicatrization effect of Plantago tomentosa extract on dogs submitted to ovariohysterectomy}

\author{
Anieli Pachla ${ }^{1}$, Fernando Silveiro Ferreira da $\mathrm{Cruz}^{2}$, Christiane de Fátima Colet $^{3}$
}

\section{Resumo}

A utilização terapêutica de plantas medicinais ocorre desde a antiguidade de forma tradicional. O estudo da cicatrização e o tratamento de feridas cutâneas são de grande importância na medicina veterinária devido à alta frequência de lesões sofridas por animais. Existem diferentes espécies de plantas que possuem propriedades cicatrizantes, dentre elas cita-se a Plantago, cujo nome popular é Tanchagem, que apresenta indicações anti-inflamatória e cicatrizante. O presente estudo teve como objetivo avaliar o efeito do extrato etanólico das folhas secas de Plantago tomentosa Lam. no processo de cicatrização pós-operatória em cães. Foram utilizadas 12 cadelas submetidas a ovariohisterectomia, divididos aleatoriamente em dois grupos de 6 animais: Grupo Controle (GC) e Grupo Tanchagem (GT). O GT apresentou melhor efetividade em relação ao GC durante um acompanhamento de quatorze dias.

Palavras chave: Plantas medicinais. Extratos. Plantago. Cicatrização de feridas. Cães.

\begin{abstract}
The therapeutic use of medicinal plants has traditionally occurred in antiquity. The study of wound healing and the treatment of cutaneous wounds are of great importance in veterinary medicine due to the high frequency of injuries suffered by animals. There are different plant species that have cicatrizing properties, among them the Plantago, whose popular name is Tanchagem, has anti-inflammatory and healing indications. The present study had as objective to evaluate the effect of the ethanol extract of the dry leaves of Plantago tomentosa Lam. in the process of postoperative cicatrization in dogs. Twelve dogs submitted to ovariohysterectomy were randomly divided into two groups of six animals: Control Group (CG) and Group Treatment (GT). The GT showed better effectiveness in relation to the CG during a fourteen-day follow-up.
\end{abstract}

Keyword: Medicinal plants, extract, Plantago, cicatrization, dogs.

\footnotetext{
${ }^{1}$ Graduação em Farmácia pela Universidade Regional do Noroeste do Estado do Rio Grande do Sul, Ijuí, Rio Grande do Sul, Brasil.

${ }^{2}$ Professor Adjunto do Departamento e Estudos Agrários da Universidade Regional do Noroeste do Estado do Rio Grande do Sul, Ijuí, Rio Grande do Sul, Brasil.

${ }^{3}$ Doutorado em Ciências Farmacêuticas pela Universidade Federal do Rio Grande do Sul. Professora Adjunta do Departamento de Ciências da Vida da Universidade Regional do Noroeste do Estado do Rio Grande do Sul, Ijuí, Rio Grande do Sul, Brasil. E-mail: chriscolet@yahoo.com.br
} 


\section{Introdução}

A utilização terapêutica de plantas medicinais ocorre desde a antiguidade de forma tradicional. Contudo, para que sejam prescritos e indicados por profissionais da saúde, necessita-se que sua eficácia e segurança sejam comprovadas por meio de estudos (BATTISTI et al., 2013). De acordo com Santos et al. (2008), a Organização Mundial de Saúde estabelece que são imprescindíveis as investigações experimentais acerca de plantas medicinais, para garantir esta eficácia e segurança terapêutica à população.

Entre as utilizações descritas para plantas medicinais destaca-se o estudo da cicatrização e o tratamento de feridas cutâneas, com importância na medicina veterinária, devido à alta frequência de lesões sofridas por animais. As feridas podem ser originadas por fatores extrínsecos, como incisões cirúrgicas, ou intrínsecos, como aquelas causadas por defeitos metabólicos ou infecções (SIMAS, 2010).

O processo de cicatrização começa logo após a lesão ou a incisão, com a finalidade de cura e pode ser dividido em três fases: inflamatória, proliferativa e de remodelação (ISAAC et al., 2010). No entanto, há a possibilidade de acelerar a cicatrização e o fechamento de lesões cutâneas com os tratamentos medicamentosos, bem como com uso de plantas medicinais (SIMAS, 2010).

Comomencionado, a busca por métodos terapêuticos medicinais com produtos a base de plantas, com a finalidade de cicatrização de lesões, tem sido objeto de investigação dos pesquisadores. Segundo Freitas et al. (2002), existem diferentes espécies de plantas que possuem propriedades cicatrizantes, dentre elas cita-se a Plantago, cujo nome popular é Tanchagem, pertencente à família Plantaginaceae. Estas plantas crescem em clima temperado ou subtropical e geralmente são utilizadas no tratamento de inflamações de boca e garganta, infecções intestinais e como agente antibacteriano (TELES; COSTA, 2014).

Os produtos derivados da espécie vegetal podem ser utilizados de forma isolada, na forma de extrato bruto, fracionado ou purificado, sendo que a importância destes deve estar relacionada à ação benéfica para a saúde dos seres humanos ou animais (FONSÊCA, 2005). No caso da Plantago, as referências citam diferentes tipos de soluções extrativas como derivados de folhas, caules, flores, sementes e raízes. Além disso, diferentes métodos extrativos são empregados para esta planta, como: maceração, decocção, infusão, turbólise, percolação em aparelho tipo Soxhlet, refluxo e extração em banho ultrassom (BRASIL, 2014).

Em relação aos metabólitos secundários produzidos pela Plantago tomentosa Lam. destacamse os alcaloides e flavonoides. No estudo de Haeffner et al. (2012), a Plantago major é descrita como antiinflamatória tendo efeito analgésico. A Plantago tomentosa LAM se assemelha a Plantago major por conter alguns metabólitos como os flavonoides (BONFIM et al., 2011).

$\mathrm{O}$ estudo e conhecimento das plantas medicinais, através de métodos experimentais, são fundamentais para o tratamento de doenças ou para o desenvolvimento de um produto novo, permitindo a associação correta entre diferentes tratamentos, obtendo protocolos ideais para cada caso clínico, reduzindo o tempo de cicatrização. Além disso, os experimentos podem auxiliar na comprovação dos efeitos das plantas utilizadas pela população (SIMAS, 2010).

Diante do exposto, o objetivo geral deste estudo foi avaliar o efeito do extrato etanólico das folhas secas de Plantago tomentosa Lam. no processo de cicatrização pós-operatória em cadelas submetidas a ovariohisterectomia.

\section{Material e Métodos}

Trata-se de um ensaio clínico contra tratamento padrão, que foi realizado no Hospital Veterinário da Universidade Regional do Noroeste do Estado do Rio Grande do Sul - UNIJUÍ, na cidade de Ijuí/RS.

Os cães foram divididos aleatoriamente em dois grupos de 6 animais: Grupo Controle (GC) e Grupo Tanchagem (GT). O GC corresponde aos animais submetidos ao tratamento com solução salina e o GT ao grupo de cães submetidos ao tratamento com extrato etanólico de Plantago tomentosa Lam. Segundo Tazima et al. (2008), a etapa de proliferação inicia-se por volta do $3^{\circ}$ dia após a lesão e ocorre de 2 a 3 semanas, dessa forma, cada grupo foi avaliado por um período de 14 dias, podendo observar o marco inicial da formação da cicatriz, etapa esta conhecida como remodelação, que tem duração indeterminada, dependendo da reorganização do colágeno de cada animal. O protocolo anestésico foi constituído por medicação pré-anestésica com acepromazina e metadona na dose de $0,03 \mathrm{mg} / \mathrm{kg}$ e $0,2 \mathrm{mg} / \mathrm{kg}$, respectivamente, por via intramuscular e posterior indução anestésica com Propofol $5 \mathrm{mg} / \mathrm{kg}$ via intravenosa. Ato contínuo, os animais sofreram intubação orotraqueal e mantidos em 
plano anestésico com Isoflurano diluído em 100\% de oxigênio em circuito semi-fechado de anestesia.

Para a avaliação da cicatrização dos cães, foram utilizados parâmetros clínicos como: estado geral do cão, dor ao toque, secreção, eritema e tamanho da lesão. A solução de tanchagem foi aplicada com o auxílio de uma gaze, sendo a quantidade aplicada determinada pelo total cobrimento da lesão, com indicação de aplicação de $12 \mathrm{em} 12$ horas. O procedimento de aplicação foi realizado pelos proprietários das cadelas e as avaliações dos animais foram realizadas pela própria pesquisadora, ciente do tratamento e da divisão dos grupos de cada animal. O acompanhamento clínico foi realizado a cada três dias, e os registros com medidas e imagens foram feitos a cada sete dias.

Para a produção do extrato foi utilizada a espécie botânica P. tomentosa Lam., coletada de forma extrativa na região noroeste do Rio Grande do Sul, Ijuí/RS, coordenadas geográficas de referência são a latitude $28^{\circ} 17^{\prime} 33^{\prime \prime}$ Sul e a longitude $53^{\circ} 30^{\prime} 06^{\prime \prime}$ Oeste. As folhas foram colhidas e selecionadas, retirando-se as partes doentes e danificadas, assim como qualquer outra parte com alteração do vegetal. A secagem foi realizada naturalmente, em temperatura ambiente e ao abrigo do sol, calor e vento, durante aproximadamente três semanas, no período de dezembro de 2015 .

A preparação do extrato foi realizada no laboratório de Farmacognosia da Universidade Regional do Noroeste do Estado do Rio Grande do Sul - UNIJUÍ, de acordo com a metodologia apresentada em Simões et al. (2004), através da maceração, na qual foi triturado $15 \mathrm{~g}$ do material vegetal em três recipientes juntamente com $150 \mathrm{~mL}$ de solvente etanol. Os recipientes de vidro foram agitados durante sete dias. Na sequência, o extrato foi filtrado em papel filtro, graduação 388, e colocado em novo recipiente de vidro e armazenado em geladeira, temperatura controlada entre 2 a $8^{\circ} \mathrm{C}$, até o momento da aplicação. A metodologia deste estudo foi adaptada de Cordeiro (2005).

A análise estatística foi realizada no programa SPSS versão 18. Realizou-se análise de teste $\mathrm{t}$ de Student para amostra dependente entre os dias $1^{\circ}$ e $7^{\circ}$, $7^{\circ}$ e $14^{\circ}$. Considerou-se $\mathrm{p}<0,05$ como significativo.

Para o desenvolvimento deste projeto, o mesmo foi submetido e aprovado pela Comissão de Ética no Uso de Animais (CEUA) da Universidade Regional do Noroeste do Estado do Rio Grande do Sul (UNIJUÍ) sob o parecer consubstanciado CEUA - 004/2016. A exsicata está disponível no Herbário com número
3501, que está cadastrado do Registro Brasileiro de Herbário.

\section{Resultados e Discussões}

O acompanhamento do processo cicatricial foi realizado em 12 cadelas, desde a incisão até a completa epitelização. No início do tratamento a evolução cicatricial foi considerada clinicamente normal, em ambos os grupos. Com isso, pode-se verificar a redução da área afetada em ambos os grupos (GC e GT). Contudo, a maioria dos proprietários relatou não seguir corretamente as indicações de tratamento, realizando as aplicações duas vezes ao dia como preconizado, mas todos informaram usar pelo menos uma vez ao dia.

O Quadro 1 apresenta os animais controle, ou seja, aqueles que tiveram o tratamento com a solução padrão. Destaca-se que os proprietários dos animais A, $\mathrm{C}$ e D afirmaram que aplicaram o tratamento correto todos os dias, o $\mathrm{B}$ relatou não seguir completamente a indicação de uso, utilizando apenas uma vez ao dia na maioria do período de tratamento. Nas cadelas E e F, foi relatado a falha da aplicação, mas em apenas uma vez durante o período de tratamento.

Dos animais deste grupo, observou-se em B a presença de secreção durante o tratamento, no $C$ observou-se eritema e no $F$ foi observada dor nos primeiros dias de tratamento. $\mathrm{O}$ animal $\mathrm{C}$ foi acompanhado por um período maior de tempo, já que a ferida apresentou edema e eritema.

Além disso, aplicou-se um questionário aos proprietários em todas as visitas, em relação à alimentação, quantidade e facilidade/dificuldade nas aplicações e estado do animal, além de verificar o tamanho da incisão. Nenhum proprietário relatou ter dificuldade de aplicar o tratamento e todas as cadelas alimentaram-se normalmente durante o tratamento.

Os resultados obtidos nas aplicações do extrato etanólico de Plantago tomentosa Lam. estão apresentados no Quadro 2. Os proprietários dos animais A, B, D e F aplicaram o tratamento correto todos os dias, no animal E foi relatado falha na aplicação do tratamento, com aplicação em alguns dias somente uma vez ao dia. O proprietário da cadela $\mathrm{C}$ relatou aplicar todo o tratamento somente uma vez ao dia.

Dos animais deste grupo, somente o $\mathrm{F}$ demonstrou dor durante o tratamento. Nenhum proprietário relatou ter dificuldade de aplicar o tratamento e todas as cadelas alimentaram-se normalmente. 
Quadro 1 - Incisão das cadelas do GC no primeiro (D1) e último dia de acompanhamento (D14).

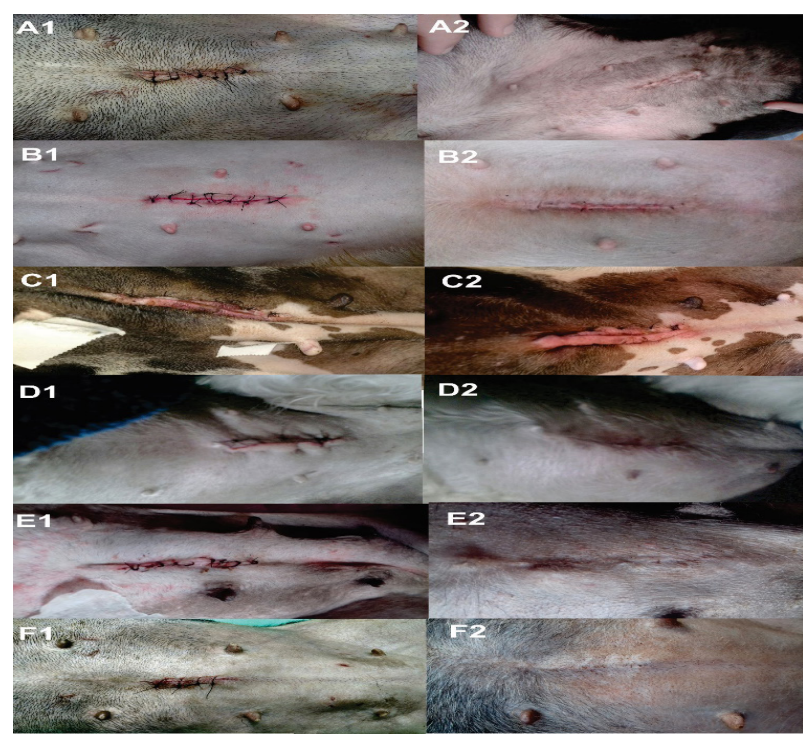

Fonte: Autores.

Quadro 2 - Incisão das cadelas do GT no primeiro (D1) e último dia de acompanhamento (D14).

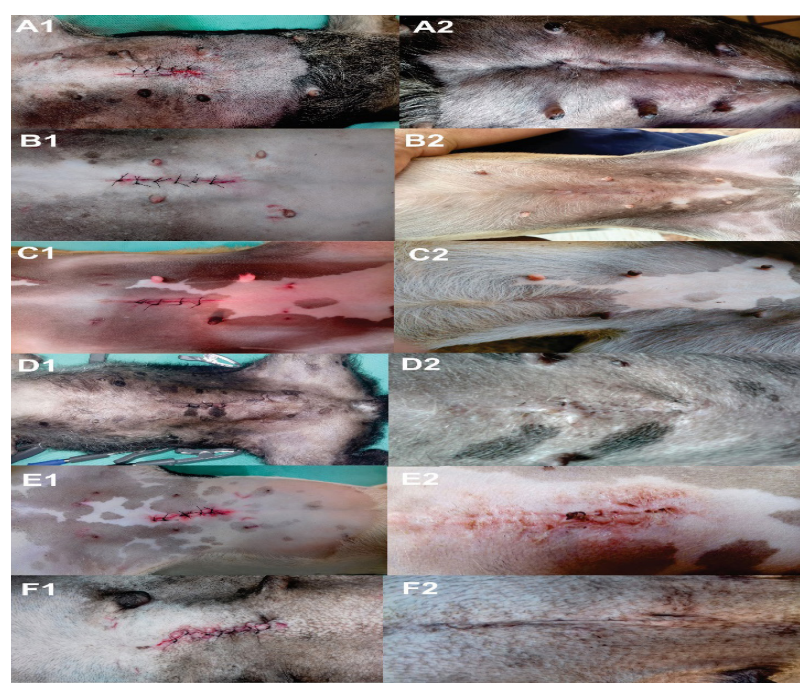

Obs: na primeira coluna está representado o dia da incisão de ambas as cadelas, na segunda coluna pode-se observar o último dia de acompanhamento.

Fonte: Autores.

A Tabela 1 apresenta as medidas das incisões, em centímetros, no primeiro, sétimo e décimo quarto dia de acompanhamento. A análise estatística dos dados referentes aos percentuais das lesões demonstrou que no GT houve significância $(0,01 ; 0,03)$ nos resultados do grupo tratamento entre os dias 01 e 07 e 07 e 14, com diminuição significativa da incisão entre os dias analisados. Por outro lado, no grupo controle, não houve significância $(0,141 ; 0,06)$ no tratamento entre os dias 01 e 07 e 07 e 14.

Tabela 1 - Medidas das incisões das cadelas nos grupos controle e Plantago tomentosa Lam. no $1^{\circ}, 7^{\circ}$ e $14^{\circ}$ dia de tratamento.

\begin{tabular}{|c|c|c|c|c|c|}
\hline Períodos & $1^{\circ} \mathrm{dia}$ & $7^{\circ} \mathrm{dia}$ & p1 & $14^{\circ} \mathrm{dia}$ & p2 \\
\hline & rupo Cont & & \multirow{7}{*}{0,141} & & \multirow{7}{*}{0,06} \\
\hline GC 1 & $4,3 \mathrm{~cm}$ & $4,0 \mathrm{~cm}$ & & $3,0 \mathrm{~cm}$ & \\
\hline $\mathrm{GC} 2$ & $5,2 \mathrm{~cm}$ & $4,2 \mathrm{~cm}$ & & $3,9 \mathrm{~cm}$ & \\
\hline $\mathrm{GC} 3$ & $4,3 \mathrm{~cm}$ & $6,6 \mathrm{~cm}$ & & $4,0 \mathrm{~cm}$ & \\
\hline $\mathrm{GC} 4$ & $3,4 \mathrm{~cm}$ & $2,7 \mathrm{~cm}$ & & $1,8 \mathrm{~cm}$ & \\
\hline GC 5 & $5,1 \mathrm{~cm}$ & $4,6 \mathrm{~cm}$ & & $3,8 \mathrm{~cm}$ & \\
\hline \multirow[t]{2}{*}{ GC6 } & $2,5 \mathrm{~cm}$ & $1,8 \mathrm{~cm}$ & & $1,0 \mathrm{~cm}$ & \\
\hline & upo Tanch & & & & \\
\hline GT 1 & $5 \mathrm{~cm}$ & $4,2 \mathrm{~cm}$ & & $3,4 \mathrm{~cm}$ & \\
\hline GT 2 & $4,7 \mathrm{~cm}$ & $3,8 \mathrm{~cm}$ & & $2,8 \mathrm{~cm}$ & \\
\hline GT 3 & $4,0 \mathrm{~cm}$ & $3,6 \mathrm{~cm}$ & \multirow{4}{*}{$0,01 *$} & $2,8 \mathrm{~cm}$ & \multirow{4}{*}{$0,03 *$} \\
\hline GT 4 & $2,7 \mathrm{~cm}$ & $2,0 \mathrm{~cm}$ & & $1,6 \mathrm{~cm}$ & \\
\hline GT 5 & $4,2 \mathrm{~cm}$ & $3,6 \mathrm{~cm}$ & & $2,7 \mathrm{~cm}$ & \\
\hline GT 6 & $5,1 \mathrm{~cm}$ & $4,6 \mathrm{~cm}$ & & $3,7 \mathrm{~cm}$ & \\
\hline
\end{tabular}

Obs: as medidas foram realizadas conforme o comprimento da incisão. Além disso, em p1 e p2 se tem a significância do tratamento. Fonte: Autores.

A comparação diária da área de epitelização, demonstrada no Quadro 1 e 2, demonstra os melhores resultados nas feridas do GT. Embora a Plantago tomentosa Lam. não tenha estudos científicos que comprovam seu efeito, pode-se perceber sua ação farmacológica nas feridas tanto pelas imagens como pelos dados estatísticos. Adotou-se no presente estudo o primeiro, sétimo e décimo quarto dia para análise dos parâmetros. Segundo Simões et al. (1986) são os dias mais significantes para o estudo do processo de reparação tecidual da pele.

Alguns estudos como Martins (2010) avaliaram o uso de plantas, como Aloe vera, na cicatrização de feridas abertas provocadas cirurgicamente em cães e concluíram que este extrato proporciona uma melhor reepitelização, estimula a migração de células epiteliais e evita a formação de crosta na ferida. No estudo de Vieira (2015), avaliou-se o efeito cicatrizante de Inga subnuda e Pseudopiptadenia contorta em feridas cirúrgicas em coelhos e concluíram que estes extratos apresentam efeito cicatrizante na ferida. Ambos os estudos atribuíram à atividade descrita a presença de 
flavonóides nas plantas. Contudo, não foram encontrados estudos específicos sobre a Plantago tomentosa Lam., em especial com metodologia como a utilizada neste estudo, embora ela seja utilizada popularmente no tratamento de enfermidades dérmicas (CRUZ; ROSARIO, 2015).

O estudo de Leite et al. (2015) avaliou o extrato metanólico da Mimusops elengi Linn. e verificou que o mesmo é eficaz na cicatrização de feridas por incisão, após 10 dias de tratamento diário em ratos. Esta planta, segundo indica o artigo, apresenta entre seus metabólitos secundários, flavonóides, saponinas, taninos, cumarinas, esteróis e compostos fenólicos. Sarmento et al. (2014) estudaram a ação do extrato etanólico bruto da Z. tuberculosada em ratos tratados por via tópica, durante 14 dias, demonstrando resultados positivos em relação à atividade cicatrizante, o artigo não apresenta metabólitos responsáveis pela ação.

Segundo Ozaki e Duarte (2006) a Sambucus nigra L. possui propriedades cicatrizantes, a Calêndula officinalis L. possui propriedades anti-inflamatórias e são plantas utilizadas em medicina veterinária, em cães e gatos. Ambas as plantas possuem flavonoides, um dos principais grupos de substâncias com atividades farmacológicas, e estes constituintes tem relação com o processo de cicatrização em feridas (VIEIRA et al., 2008). Destacase que este constituinte também está presente na Plantago tomentosa Lam. e pode ser responsável pelo efeito cicatrizante encontrado.

São os metabolismos secundários, os responsáveis pela ação biológica diretamente contra patógenos (SILVA, 2013) e entre eles, os flavonoides estimulam a cicatrização agindo como antioxidantes, além de possuir atividade antimicrobiana, ação anti-inflamatória, analgésica e regenerativa (JANNING et al., 2011). Os flavonoides são substâncias orgânicas, nitrogenadas, não proteicas e não se apresentam em grupos homogêneos (FONSÊCA, 2005). A atividade farmacológica dos flavonoides se deve a forma estrutural característica, o composto tricíclico, ou seja, estas propriedades estão relacionadas com a presença de radicais livres nos seus anéis (VIEIRA et al., 2008). Desta forma, pode-se atribuir a atividade avaliada no presente estudo a este composto, o que justifica sua atividade farmacológica.

Segundo Cruz e Rosário (2015), os princípios ativos mais comuns da Plantago tomentosa Lam. são os alcaloides e flavonoides. No estudo de Haeffner et al. (2012), a Plantago major é descrita como antiinflamatória, tendo efeito analgésico. A Plantago tomentosa LAM se assemelha a Plantago major por conter alguns metabólitos, como os flavonoides (BONFIM et al., 2011).

Para Piriz et al. (2015) referente ao uso popular de plantas medicinais na cicatrização de feridas em enfermagem, através de uma entrevista semiestruturada gravada, o autor analisou farmacologicamente a ação dos extratos hidroalcóolicos de Plantago australis comprovando a ação analgésica e anti-inflamatória. No mesmo estudo os autores avaliaram a espécie Plantago ovata demonstrando ação antisséptica e anti-inflamatória e, ainda, o autor relata que os polifenóis, extraídos de folhas e sementes de Plantago major apresentaram efeitos cicatrizantes de feridas em enfermagem.

Algumas limitações deste estudo foram as diferenças quanto a idade e o porte do animal, o fio utilizado na sutura, que variou de acordo com o porte do animal, embora tenha sido de Nylon monofilamento para todos os animais, e a aplicação do tratamento ter sido realizado pelo proprietário que nem sempre manteve a adesão ao tratamento.

A opção por Plantago tomentosa Lam. em detrimento da Plantago major, que é mais conhecida e estudada, está relacionada ao predomínio da primeira na região noroeste do Rio Grande do Sul. E considerando não haverem estudos científicos referente a Plantago tomentosa Lam. buscando comprovar seus efeitos em cicatrização cutânea, sendo esse o foco do presente trabalho.

\section{Conclusão}

O emprego do extrato etanólico de Plantago tomentosa Lam. no processo cicatricial de incisões cirúrgicas em cães demonstrou, neste estudo, efeito significativo na cicatrização da pele, comprovando o seu uso popular neste efeito. Desta forma, o emprego do extrato pode ser uma alternativa em Medicina Veterinária, pelo custo reduzido, visto os resultados significativos quanto ao efeito terapêutico.

Ainda há muito a evoluir quanto à avaliação da eficácia, segurança e qualidade na utilização de Plantago tomentosa Lam, destacando-se, assim, a importância e a necessidade da realização de novos testes com o extrato etanólico das folhas secas de Plantago tomentosa Lam que possam contribuir com esta pesquisa, comprovando a ação cicatricial dos flavonoides, presentes nesta planta, além de garantir à população o uso de produtos naturais com mais segurança. 


\section{Referências}

BATTISTI, C.; GARLET, T. M. B.; ESSI, L.; HORBACH, R. K.; ANDRADE, A.; BADKE, M. R. Plantas medicinais utilizadas no município de Palmeira das Missões, RS, Brasil. Revista Brasileira de Biociências, Porto Alegre, v. 11, n. 3, p. 338-348, jul./set. 2013.

BONFIM, F. P. G.; HONÓRIO, I. C. G.; CASALI, V. W. D.; FONSECA, M. C. M.; MANTOVANIALVARENGA, E.; ANDRADE, F. M. C.; PEREIRA, A. J.; GONÇALVES, M. G. Potencial alelopático de extratos aquosos de Melissa officinalis L. e Mentha $x$ villosa $\mathrm{L}$. na germinação e vigor de sementes de Plantago major L. Revista Brasileira de Plantas Medicinais, Botucatu, v. 13, n. spe. 2011. Disponível em: $<$ http://www.scielo.br/scielo.php?script=sci_artte $\mathrm{xt} \& \mathrm{pid}=\mathrm{S} 1516-05722011000500010>$. Acesso em: 16 nov. 2016.

BRASIL. Monografia da espécie Plantago major L. (Tanchagem). Brasília: Ministério da Saúde; Anvisa, 2014. Disponível em: <http://u.saude.gov.br/images/ pdf/2014/novembro/25/Vers--o-cp-Plantago-major. pdf>. Acesso em: 14 out. 2015.

CORDEIRO, C. H. G. Atividade biológica de gel dentifrício e enxaguatório bucal contendo extratos vegetais. Araraquara: Universidade Estadual Paulista, 2005 .

CRUZ, T. K. T; ROSARIO, J. M. Z. Plantago tomentosa L. uma revisão bibliográfica. In: SEMINÁRIO DE INICIAÇÃO CIENTÍFICA DA UNIJUÍ, 23., 2015, Ijuí. Anais... Ijuí: UNIJUÍ, 2015. Disponível em: $<$ https://www.revistas.unijui.edu.br/index.php/ salaoconhecimento/article/viewFile/4979/4165>. Acesso em: 17 nov. 2016.

FONSÊCA, S. G. C. Farmacotécnica de fitoterápicos. Fortaleza: Departamento de Farmácia - UFC, 2005.

FREITAS, A. G.; COSTA, V.; FARIAS, E. T.; LIMA, M. C. A.; SOUSA, I. A.; XIMENES, E. A. Atividade antiestafilocócica do Plantago major L. Revista Brasileira de Farmacognosia, Maringá, v. 12, suppl. 1, p. 64-65, 2002.
HAEFFNER, R.; HECK, R. M.; CEOLIN, T.; JARDIM, V. M. R.; BARBIERI, R. L. Plantas medicinais utilizadas para o alívio da dor pelos agricultores ecológicos do Sul do Brasil. Revista Eletrônica de Enfermagem, Goiânia, v. 14, n. 3, p. 596-602, jul./set. 2012.

ISAAC, C.; LADEIRA, P. R. S.; RÊGO, F. M. P.; AlbunAte, J. C. B.; FERREIRA, M. C. Processo de cura das feridas: cicatrização fisiológica. Revista de Medicina, São Paulo, v. 89, n. 3-4 p. 125-31, jul./dez. 2010.

JANNING, D.; ALBUQUERQUE, C. A. C.; BARAUANA, S. C. Avaliação preliminar do extrato hidroalcoólico de Tabernaemontana catharinensis no processo de cicatrização de feridas em pele de ratos (Rattus Norvegicus). Revista Eletrônica de Farmácia, Goiânia, v. 8, p. 53-64, 2011.

LEITE, N. S.; LIMA, A. P.; ARAÚJO-NETO, V.; ESTEVAM, C. S.; PANTALEÃO, S. M.; CAMARGO, E. A.; FERNANDES, R. P. M.; COSTA, S. K. P.; MUSCARÁ, M. N.; THOMAZZI, S. M. Avaliação das atividades cicatrizante, anti-inflamatória tópica e antioxidante do extrato etanólico da Sideroxylon obtusifolium (quixabeira). Revista Brasileira de Plantas Medicinais, Campinas, v. 17, n. 1, p. 164-170, 2015 .

MARTINS, J. M. O uso da babosa (Aloe vera) na reparação de feridas abertas provocadas cirurgicamente em cães. 2010. Monografia (Bacharelado em Medicina Veterinária) - Universidade Federal de Campina Grande, Centro de Saúde e Tecnologia Rural Campus de Patos- PB, Patos, 2010.

OZAKI, A. T.; DUARTE, P. C. Fitoterápicos utilizados na medicina veterinária, em cães e gatos. Infarma, Brasília, v. 18, n. 11-12, 2006.

PIRIZ, M.A.; ROESE, A.; LOPES, C. V.; SILVA, M. M.; HECK, R. M.; BARIERI, R. L. Uso popular de plantas medicinais na cicatrização de feridas: implicações para a enfermagem. Revista de Enfermagem UERJ, Rio de Janeiro, v. 23, n. 5, p. 674-9, set./out. 2015. 
SANTOS, M. R. A.; LIMA, M. R.; FERREIRA, M. G. R. Uso de plantas medicinais pela população de Ariquemes, em Rondônia. Horticultura Brasileira, Vitória da Conquista, v. 26, p. 244-250, 2008. Disponível em: <http://www.scielo.br/pdf/hb/ v26n2/23.pdf $>$. Acesso em: 17 out. 2015.

SARMENTO, P. A.; ATAÍDE, T. R.; BARBOSA, A. P. F.; ARAÚJO JUNIOR, J. X.; LÚCIO, I. M. L.; BASTOS, M. L. A. Avaliação do extrato da Zeyheria tubeculosa na perspectiva de um produto para cicatrização de feridas. Revista Latino-Americana de Enfermagem, Ribeirão Preto, v. 22, n. 1, p. 165-72, jan./fev. 2014.

SILVA, C. M. A. Metabólitos secundários de plantas do semiárido de Pernambuco: uma inovação no controle de fitopatógenos. 2013. Dissertação (Mestrado em Bioquímica e Fisiologia) - Universidade Federal de Pernambuco, Recife, 2013.

SIMAS, S. M. O tratamento de feridas cutâneas em cães e gatos. 2010. 104 f. Trabalho de Conclusão de Curso (Graduação em Medicina Veterinária) - Universidade Federal do Rio Grande do Sul, Faculdade de Veterinária, Porto Alegre, 2010. Disponível em: $<$ https://www.lume.ufrgs.br/bitstream/ handle $/ 10183 / 39023 / 000793034$.pdf? sequence $=1>$ Acesso em: 20 out. 2015.

SIMÕES, C. M. O.; SCHENKEL, E. P.; GOSMANN, G.; MELLO, J. C. P.; MENTZ, L. A.; PETROVICK, P. R. Farmacognosia: da planta ao medicamento. 5.ed. Porto Alegre: UFRGS; Florianópolis: UFSC, 2004.

SIMÕES, M. J.; CABRAL, A. C. V.; BOYACIYAN, K.; KULAY JUNIOR, I.; SASSO, W. S. Aspectos ultraestruturais dos fibroblastos e dos macrófagos durante o processo de reparação da pele de ratos. Revista Paulista de Medicina, São Paulo, v. 104, n. 3, p. 132-5, 1986.

TAZIMA, M. F. G. S.; VICENTE, Y. A. M. V. A.; MORIYA, T. Biologia da ferida e cicatrização. Medicina, Ribeirão Preto, v. 41, n. 3, p. 259-64, 2008. Disponível em: <http://revista.fmrp.usp.br/2008/ VOL41N3/SIMP_2Biologia_ferida_cicatrizacao. pdf $>$. Acesso em: 10 fev. 2016.
TELES, D. G.; COSTA, M. M. Estudo da ação antimicrobiana conjunta de extratos aquosos de Tansagem (Plantago major I., Plantaginacea) e Romã (Plunica granatum I., Punicaceae) e interferência dos mesmos na ação da amoxicilina in vitro. Revista Brasileira de Plantas Medicinais, Campinas, v. 16, n. 2, supl. 1, p. 323-328, 2014.

VIEIRA, A. P.; SANTOS, N. R.; BORGES, J. H. S.; VICENZI, M. P. A.; SCHMITZ, W. O. Ação dos flavonoides na cicatrização por segunda intenção em feridas limpas induzidas cirurgicamente em ratos Wistar. Semina: Ciências Biológicas e da Saúde, Londrina, v. 29, n. 1, p. 65-74, jan./jun. 2008.

VIEIRA, G. T. Avaliação do efeito cicatrizante de Inga subnuda e Pseudopiptadenia cortorta em feridas cirúrgicas em coelhos. 2015. Tese (Doutorado em Bioquímica Aplicada) - Universidade Federal de Viçosa, Viçosa.

Recebido em: 02 fev. 2017

Aceito em: 05 set. 2017 
Pachla, A. ;et al. 